\title{
Examples of Role of Metrology in Materials Science \& Engineering
}

Received: December 30, 2015; Accepted: January 02, 2016; Published: January 05, 2016

Metrology is fundamental to all biological, physical and environmental sciences, engineering, and medicine. Standards are required for all measurements. While manufacturing depends on research and development, research and development would not be possible without reliable experimental data, the analytical instrumentation tools and methods to obtain authentic experimental data and the methods to calibrate against standards. It is within this context that The Journal of Scientific and Industrial Metrology is timely and relevant.

The demands on metrology have grown over the years. This is particularly the situation in a variety of material scenarios. At the macro level, these scenarios include examples such as alloys, bandgap engineered structures, biomaterials, ceramics, composite materials, coatings, electronic materials, liquid crystals, magnetic materials, metals, metamaterials, nanomaterials, optical materials, polymers, semiconductors, smart materials, superconductors etc. The ability to tailor materials, processes, performance and structure, for desired applications, requires pre-knowledge of the material properties. At the device and systems level, applications of metrology include detectors, drug delivery systems, energy sources, filters, imagers, lasers, process control systems, sensors, waveguides etc. The influence of defects and dislocations on properties becomes significant especially when the structures are scaled from micron to the nanoscale. The enormous progress that has been made in twodimensional materials such as graphene [1], transition metal dichalcogenides/layered metal dichalcogenides (TMDC/LMDC) [2] such as molybdenum disulfide [3], tungsten disulfide [4] and related materials, makes it imperative that the properties of these materials be available to advance the science and applications.

Fundamental research, both experimental and theoretical, requires metrology. There has been a tremendous growth in computational tools and instrumentation methods; the accuracy, repeatability and reproducibility of the measured data has improved significantly. Throughout the globe, industry, research laboratories and universities have been developing and improving methods for reliable and accurate data that represents the measurement of the physical quantity.

Case studies of such research activities include the development of pyrometry. Pyrometers [5] are the instruments of choice for noncontact in situ temperature measurements in materials processing. Pyrometers measure the amount of radiation emitted from a material within a narrow wavelength window. The ratio of the emitted radiation by the material to that of a blackbody

\section{Nuggehalli M Ravindra}

Interdisciplinary Program in Materials Science \& Engineering, New Jersey Institute of Technology, Newark, NJ, USA

Corresponding author: Nuggehalli M Ravindra

New Jersey Institute of Technology, USA. n.m.ravindra@njit.edu

\section{Tel: 9735963278}

Citation: Ravindra NM.Examples of Role of Metrology in Materials Science \& Engineering. J Sci Ind Metrol. 2015 1:1.

under the same conditions of temperature, wavelength, angle of incidence, and direction of polarization is referred to as emissivity. Emissivity of materials is a complicated function of both temperature and wavelength. In addition, it is also sensitive to surface roughness. Emissivity is obtained from simultaneous measurements of hemispherical transmittance, reflectance and absorptance. The related fundamental optical constants, refractive index and extinction coefficient, and their wavelength dependence in the infrared range of wavelengths, are required in a variety of process monitoring applications. The knowledge of these fundamental optical constants has significant implications in developing non-contact sensors as well as in developing measurement techniques such as spectroscopic ellipsometry [6] and time resolved reflectivity [7].

Another example is thermoelectrics [8]. With their ability to recover waste heat and convert it into useful electricity, thermoelectric (TE) materials are promising candidates to achieve the challenge to reduce energy wastage. Most important applications of TE materials are in coolers and power generators to convert thermal energy into electrical energy and vice versa. A dimensionless quantity, $\mathrm{ZT}\left(\mathrm{ZT}=\mathrm{S}^{2} \sigma \mathrm{T} / \mathrm{K}\right)$, and the power factor $\left(S^{2} \sigma\right)$, are used to evaluate the performance of thermoelectric (TE) materials; $\mathrm{S}$ is the Seebeck coefficient (or thermoelectric power), $\sigma$ is the electrical conductivity, $\mathrm{k}$ is the thermal conductivity and $\mathrm{T}$ is the temperature (in K) [9]. Measurements of $\mathrm{S}$ are not always easily available for a number of materials. 
Research on biomaterials [10] and related topics is on an exponential rise. This has been catalyzed by increased human life expectancy and, simultaneously, the need to improve the quality of life. Drug delivery systems are being developed to address a variety of ailments and diseases. These systems will require complete understanding of diffusion of drug actives, in the form of molecules through materials as function of their structure and morphology [11].
With the global emphasis now on "Material is the Device" [12], metrology will play a key role in realizing this goal. The need for an infrastructure and a platform that provides access to standards is critical. By bringing together research that is performed by researchers, scientists and engineers, throughout the world, The Journal of Scientific and Industrial Metrology expects to provide that infrastructure. On behalf of the Publishers and the Editorial Board, I invite authors to submit their manuscript for consideration for publication to the Journal. 


\section{References}

1 Chen Y, Sun J, Gao J, Du F, Han Q, et al. (2015) Growing uniform graphene disks and films on molten glass for heating devices and cell culture. Advanced Materials 27: 7839-7846.

2 Ye Y, Wong Z J, Lu X, Ni X, Zhu H, et al. (2015) Monolayer excitonic laser. Nature Photonics 9: 733-737.

3 Butler S Z, Hollen S M, Cao L, Cui Y, Gupta J A, et al. (2013) Progress, challenges and opportunities in two-dimensional materials beyond graphene. ACS NANO 7: 2898-2926.

4 Wu K, Zhang X, Wang J, Li X, Chen J (2015) Two-dimensional nanomaterial tungsten disulfide $\left(\mathrm{WS}_{2}\right)$ as saturable absorber for mode-locked laser near $1550 \mathrm{~nm}$.

5 Grabas B (2016) Pyrometric temperature measurements with a miniature cavity used as a blackbody in calorimetric method for determining absorbed laser energy. Experimental thermal and fluid science 74:100-109.

6 Jellison Jr G E, Aytug T, Lupini A R, Paranthaman M P, Joshi P C (2015) Optical properties of a nanostructured glass-based film using spectroscopic ellipsometry. Thin Solid Films.

7 Kumada T, Akagi H, Itakura R, Otobe T, Yokoyama A (2014)
Femtosecond laser ablation dynamics of fused silica extracted from oscillation of time-resolved reflectivity. J. Applied Physics 115: 103504-9.

8 Jariwala B, Shah D, Ravindra N M (2015) Transport property measurements in doped $\mathrm{Bi}_{2} \mathrm{Te}_{3}$ single crystals obtained via zone melting method. J. Electronic Materials 44: 1509-1516.

9 Kim S I, Lee K H, Mun H A, Kim H S, Hwang S W, et al. (2015) Dense dislocation arrays embedded in grain boundaries for highperformance bulk thermoelectrics. Science 348: 109-114.

10 Hedayati R, Sadighi M, Mohammadi-Aghdam M, Zadpoor A A (2016) Mechanical properties of regular porous biomaterials made from truncated cube repeating unit cells: Analytical solutions and computational methods. Materials Science and Engineering C 60: 163-183.

11 Xie M, Li Y, Zhao Z, Chen A, Li J, et al. (2015) Development of silk fibroin-derived nanofibrous drug delivery system in supercritical $\mathrm{CO}_{2}$, Materials Letters.

12 Gimlett J (2015) Materials for transduction (MATRIX) Defense Advanced Research Projects Agency. 